\title{
INDICADORES ANTROPOMÉTRICOS E FISIOLÓGICOS DE UMA AMOSTRA DE ATLETAS BRASILEIROS DE RUGBY
}

\author{
MS. PAULO VICTOR MEZZAROBA \\ Programa de Pós-Graduação Associado em Educação Física, \\ Universidade Estadual de Maringá/Universidade \\ Estadual de Londrina (Maringá - Paraná - Brasil) \\ E-mail: paulomezzaroba@hotmail.com
}

\section{GRAD. CAMILA DE FÁTIMA TRINDADE}

Curso de Educação Física, Departamento de Educação Física, Centro de Ciências da Saúde, Universidade Estadual de Maringá

(Maringá - Paraná - Brasil)

E-mail: camisista@hotmail.com

\section{DRA. FABIANA ANDRADE MACHADO}

Departamento de Educação Física, Centro de Ciências da Saúde, Universidade Estadual de Maringá; Programa de Pós-Graduação Associado em Educação Física, Universidade Estadual de Maringá/

Universidade Estadual de Londrina (Maringá - Paraná - Brasil)

E-mail: famachado_uem@hotmail.com

\begin{abstract}
RESUMO
Verificou-se parâmetros fisiológicos e antropométricos de jogadores de rugby. Quinze atletas, agrupados de acordo com a posição de jogo: forwards (GF) e backs (GB) realizaram avaliação antropométrica e teste de potência anaeróbia com análise do lactato sanguíneo, frequência cardíaca e percepção subjetiva de esforço. O teste $t$ de Student mostrou diferenças entre grupos para as variáveis antropométricas, em especial massa corporal ( $G F=102,6 \pm 1$ 6, $0 \mathrm{~kg}$; $G B=80,0 \pm 6,8 \mathrm{~kg}$ ) e massa gorda ( $G F=28,0 \pm 10,6 \mathrm{~kg} ; G B=13,8 \pm 5,6 \mathrm{~kg}$ ), mas não para as variáveis mensuradas no teste de potência. Além disso, o teste de correlação de Pearson mostrou influência negativa da massa gorda no tempo de teste para $G F(r=0,73)$ e positiva da massa isenta de gordura na potência para $G B(r=0,80)$, ressaltando que a composição corporal influencia a performance no rugby, e deve ser utilizada juntamente a parâmetros fisiológicos para discriminar posições nesta modalidade.
\end{abstract}

PALAVRAS-CHAVE: Metabolismo energético; lactato; composição corporal; corrida. 
Orugby é um esporte de forte tradição e está presente em mais de 100 países (MEIR; HALLIDAY, 2005); porém, no Brasil, ainda faltam investimentos e divulgação desta modalidade. Um dos objetivos da Confederação Brasileira de Rugby (20 I I) é modificar esse panorama até 2016, ano em que o rugby retorna como esporte olímpico, e os Jogos Olímpicos serão realizados no Brasil (COMITE, 2009).

A literatura internacional aborda vários aspectos desta modalidade, desde perfil de jogadores (CREWTHER et al., 2009; ATKINS, 2006; GABBETT, 2000) e comparações de posições de jogo (MEIR et al., 200 I; BREWER; DAVIS; KEAR, 1994), até análises de partidas (MEIR; HALLIDAY, 2005; COUTTS; REABURN; ABT, 2003; DUTHIE; PYNE; HOOPER, 2003). No entanto, existe uma carência de estudos com atletas e equipes brasileiras, que em maioria são amadoras e disputam campeonatos estaduais e nacionais no Brasil.

Os estudos de investigação das demandas fisiológicas do rugby o descrevem como uma modalidade intermitente, em que as atividades de baixa intensidade são efetuadas na maior parte do jogo, porém, o sucesso das jogadas depende dos esporádicos trabalhos de alta intensidade realizados (GABBETT, 2005; GABBETT, 2002), exigindo dos atletas uma combinação de força, flexibilidade, capacidade aeróbia e, em especial, potência anaeróbia (COUTTS; REABURN; ABT, 2003).

A equipe de rubgy é composta por 15 jogadores divididos em duas principais formas de posicionamento: oito são os backs, considerados os jogadores que carregam a bola, e sete são os forwards, considerados os jogadores responsáveis por ganhar a posse de bola (DUTHIE; PYNE; HOOPER, 2003). Estas posições demandam diferentes necessidades fisiológicas e até mesmo antropométricas, sendo os forwards usualmente mais altos e mais pesados, com alta necessidade de força e potência (SCOTT et al., 2003) e os backs mais rápidos, ágeis e com maior demanda da capacidade aeróbia por permanecerem mais tempo em corrida livre (MEIR; ARTHUR; FORREST, 1993).

Diante disso, o objetivo deste estudo foi verificar alguns indicadores antropométricos e fisiológicos em teste de potência anaeróbia de uma amostra de atletas brasileiros de rugby, comparando essas características entre as posições backs e forwards.

\section{MATERIAIS E MÉTODOS}

SUJEITOS

A amostra foi composta por 15 atletas de rugby do gênero masculino, titulares de uma equipe amadora da modalidade, participantes de eventos nacionais, campeões 
paranaenses de 20 I 0, campeões da Taça Paraná de 201 I e dos Jogos Abertos deste mesmo estado em 2012. Os sujeitos estavam inseridos em treinamento sistematizado da modalidade há 3,4 \pm I,2 anos. A amostra foi dividida em dois grupos, de acordo com a posição de jogo específica da modalidade: grupo dos forwards $(\mathrm{GF}, \mathrm{n}=8)$ e grupo dos backs ( $\mathrm{GB}, \mathrm{n}=7)$. Todos os sujeitos estavam cientes dos protocolos do estudo e assinaram o termo de consentimento livre e esclarecido. $\bigcirc$ estudo foi aprovado pelo comitê de ética em pesquisa local (\# |67/20 I0).

\section{PROCEDIMENTOS}

As avaliações ocorreram no período máximo de uma semana. Em laboratório foram realizadas as avaliações antropométricas e de composição corporal, e em campo de grama descoberto com temperatura ambiente média de $29 \pm 1{ }^{\circ} \mathrm{C}$, o teste físico. Os sujeitos foram orientados a comparecerem bem hidratados e alimentados em um período mínimo de duas horas após a última refeição, vestindo roupas leves e apropriadas à realização de esforços físicos, além da chuteira específica da modalidade. Também foi solicitado que evitassem exercícios físicos intensos e o consumo de bebidas alcoólicas, cafeinadas ou energéticas nas 24 horas anteriores aos testes.

\section{ANTROPOMETRIA}

Previamente ao início dos testes físicos foram realizadas as avaliações antropométricas e de composição corporal. Todas as medidas foram realizadas por um único avaliador, sendo utilizado o valor médio de três aferições para minimizar possíveis erros. Procedimentos padronizados foram utilizados para as medidas referentes à massa corporal, estatura, circunferências de peitoral, abdome, cintura, quadril, coxa e panturrilha direita, dobras cutâneas das regiões tricipital, subescapular, peitoral, abdominal, coxa média, suprailíaca e axilar média.

A partir das variáveis mensuradas, foram calculados o índice de massa corporal (IMC) e a relação cintura quadril (RCQ), a densidade corporal (DC) foi determinada a partir do somatório das sete dobras cutâneas mensuradas (mm) e idade (anos), utilizando-se a equação abaixo (JACKSON; POLLOCK, 1978):

DC $\left(\mathrm{g} \cdot \mathrm{mL}^{-1}\right)=$ । , I 200000 - [0,00043499 × (soma das sete dobras cutâneas) $]+\left[0,00000055 \times(\text { soma das sete dobras cutâneas })^{2}\right]-(0,00028826 \times$ idade $)$.

Em seguida, o percentual de gordura (\%G) foi calculado a partir do DC utilizando-se a equação de Siri (|96|): 


$$
\% \mathrm{G}=(495 / \mathrm{DC})-450
$$

A massa isenta de gordura (MIG) foi calculada a partir da massa corporal total e massa gorda.

\section{TESTE DE POTÊNCIA ANAERÓBIA}

A potência anaeróbia foi determinada a partir do running anaerobic-based sprint test (RAST) (ZACHAROGIANNIS; PARADISIS; TZIORTZIS, 2004). O RAST é um teste de fácil aplicação, confiável e validado para avaliação da potência anaeróbia, que pode fornecer uma estimativa dos determinantes energéticos e neuromusculares do desempenho anaeróbio máximo. Além disso, este teste é apropriado para atividades que têm a corrida como principal forma de locomoção (ZAGATTO; BECK; GOBATTO, 2009).

Após 10 minutos de aquecimento, os sujeitos foram encaminhados à área previamente preparada em campo de grama para realização do teste, com marcações de 35 m e 5 m de área de frenagem em cada extremidade. $\bigcirc$ protocolo realizado consistiu na execução de seis desempenhos máximos de corrida na distância determinada, com intervalo de 10 segundos entre cada uma delas. $\bigcirc$ tempo correspondente a cada um dos desempenhos, bem como o tempo de intervalo, foi obtido com o auxílio de um cronômetro, por um avaliador experiente. Estímulo verbal foi utilizado em todos os testes para manutenção do esforço máximo.

Com o tempo total $(T)$ em segundos e a massa corporal em quilogramas de cada sujeito, foi possível calcular a potência anaeróbia (PAn) obtida em cada desempenho, a potência média $\left(P A n_{\text {med }}\right)$, a potência mínima $\left(P A n_{\text {min }}\right)$ e máxima $\left(P A n_{\text {max }}\right)$ dos desempenhos, e o índice de fadiga absoluto (IFI) e relativo (IF2) a partir das seguintes fórmulas (ZACHAROGIANNIS; PARADISIS; TZIORTZIS, 2004):

$$
\begin{aligned}
& \text { PAn }(W)=\text { Massa corporal }(\mathrm{kg}) \times(35)^{2} / \mathrm{T}^{3} \\
& \mathrm{PAn}_{\max }(\mathrm{W})=\text { maior potência alcançada nos seis desempenhos } \\
& \mathrm{PAn}_{\min }(W)=\text { menor potência alcançada nos seis desempenhos } \\
& \left.\mathrm{PAn}_{\text {med }}(\mathrm{W})=\text { (soma das } 6 \mathrm{PAn}\right) / 6 \\
& |F|(W / s)=\left(P A n_{\text {max }}-P A n_{\text {min }}\right) / T \\
& \text { IF2 }(\%)=\left[\left(P A n_{\text {max }}-P A n_{\text {min }}\right) / P A n_{\text {max }}\right] \times 100
\end{aligned}
$$

Foram coletadas amostras de sangue $(25 \mu \mathrm{l})$ do lóbulo da orelha dos atletas nos momentos pré, pós-teste, após três e cinco minutos do término. $\bigcirc$ sangue foi acondicionado em tubos tipo Eppendorff contendo 50 $\mu$ l de fluoreto de sódio ( $\mathrm{NaF}$ ) 
a I \% e analisadas em equipamento eletroquímico (YSI 2300 STAT, Ohio - USA) para determinação das concentrações pico de lactato sanguíneo ( Lac $_{\text {pico }}$ ), selecionando-se o maior valor obtido em uma das três coletas pós-teste.

A frequência cardíaca (FC) e a percepção subjetiva de esforço (PSE) foram monitoradas durante o teste utilizando-se um monitor cardíaco (Polar RS800) e uma escala de Borg de 6 a 20 pontos (BORG, 1982) para determinação da FC pico e PSE pico.

\section{ANÁLISE ESTATÍSTICA}

Os dados estão apresentados em média \pm desvio padrão (DP) para todas as variáveis mensuradas. Para a análise dos dados foi utilizado o programa estatístico Statistical Package for the Social Sciences (SPSS Inc. USA) versão 17.0. A normalidade de todas as variáveis do estudo foi confirmada pelo teste "W" de Shapiro-Wilk (P > 0,05). O teste t de Student para amostras não pareadas foi utilizado para comparação das variáveis entre os grupos e o teste de Pearson foi utilizado para verificar a correlação das variáveis antropométricas, de composição corporal, físicas e fisiológicas de cada grupo, o nível de significância adotado foi de $P<0,05$.

\section{RESULTADOS}

A Tabela I apresenta os valores médios \pm DP das variáveis antropométricas e descritivas da amostra total (AT), do grupo de forwards (GF) e do grupo de backs (GB). Houve diferença estatisticamente significante para todas as variáveis, exceto para estatura e relação cintura quadril (RCQ) entre os grupos.

Tabela I. Valores médios \pm desvio-padrão das características descritivas, variáveis antropométricas e de composição corporal da amostra total (AT) e dos grupos de forwards (GF) e backs (GB): massa corporal (MC), estatura, índice de massa corporal (IMC), circunferência de cintura (CC), circunferência de abdome (CA), relação cintura quadril (RCQ), circunferência de peitoral (CP), circunferência de coxa (CCoxa), circunferência de panturrilha (CPant), percentual de gordura (\%G), massa gorda (Mgorda) e massa isenta de gordura (MIG)

\begin{tabular}{ccccc}
\hline Variáveis & AT $(\mathrm{n}=15)$ & $\mathrm{GF}(\mathrm{n}=8)$ & $\mathrm{GB}(\mathrm{n}=7)$ & $\mathrm{P}$ \\
\hline Idade $(\mathrm{anos})$ & $22,5 \pm 3,3$ & $23,7 \pm 3,2$ & $21,0 \pm 2,9^{*}$ & 0,108 \\
$\mathrm{MC}(\mathrm{kg})$ & $92,1 \pm 16,8$ & $102,6 \pm 16,0$ & $80,0 \pm 6,8^{*}$ & 0,005 \\
Estatura $(\mathrm{cm})$ & $178,0 \pm 5,0$ & $181,0 \pm 5,0$ & $176,0 \pm 7,0$ & $0,88 \mid$ \\
IMC $\left(\mathrm{kg} / \mathrm{m}^{2}\right)$ & $28,8 \pm 4,6$ & $31,4 \pm 4,5$ & $25,9 \pm 2,5^{*}$ & 0,013 \\
$\mathrm{CC}(\mathrm{cm})$ & $88,6 \pm 8,2$ & $94,0 \pm 6,8$ & $82,4 \pm 4,4^{*}$ & 0,002 \\
\hline
\end{tabular}




\begin{tabular}{ccccc}
\hline Variáveis & AT $(\mathrm{n}=15)$ & GF $(\mathrm{n}=8)$ & $\mathrm{GB}(\mathrm{n}=7)$ & $\mathrm{P}$ \\
\hline RCQ & $0,8 \pm 0,02$ & $0,8 \pm 0,02$ & $0,8 \pm 0,02$ & 0,242 \\
CA $(\mathrm{cm})$ & $92,4 \pm 12,5$ & $99,9 \pm 11,8$ & $83,9 \pm 6,4^{*}$ & 0,007 \\
CP $(\mathrm{cm})$ & $103,9 \pm 8,1$ & $108,5 \pm 7,5$ & $98,7 \pm 5,1^{*}$ & 0,012 \\
CCoxa $(\mathrm{cm})$ & $64,0 \pm 6,1$ & $68,1 \pm 4,6$ & $59,2 \pm 3,4^{*}$ & 0,001 \\
CPant $(\mathrm{cm})$ & $41,2 \pm 3,2$ & $43,1 \pm 3,2$ & $39,0 \pm 1,5^{*}$ & 0,009 \\
$\% G$ & $22,1 \pm 7,9$ & $26,2 \pm 6,6$ & $17,0 \pm 6,3^{*}$ & 0,014 \\
Mgorda $(\mathrm{kg})$ & $21,4 \pm 11,1$ & $28,0 \pm 10,6$ & $13,8 \pm 5,6^{*}$ & 0,007 \\
MIG $(\mathrm{kg})$ & $70,7 \pm 7,2$ & $74,6 \pm 6,4$ & $66,3 \pm 5,6^{*}$ & 0,020 \\
\hline
\end{tabular}

*p $<0,05$ em relação ao GF

A Tabela 2 apresenta os valores médios \pm DP das variáveis fisiológicas relacionadas ao teste de potência RAST da amostra total (AT), do grupo de forwards (GF) e do grupo de backs (GB). Não houve diferença estatisticamente significante entre os dois grupos.

Tabela 2. Valores médios \pm desvio-padrão das variáveis relacionadas ao teste de RAST da amostra total (AT) e dos grupos de forwards (GF) e backs (GB): potência anaeróbia média $\left(P A n_{\text {med }}\right)$, potência anaeróbia máxima $\left(P A n_{\max }\right)$, tempo total de teste $(T)$, índice de fadiga absoluto (IFI) e relativo (IF2), pico de lactato ( $\mathrm{Lac}_{\text {pico }}$ ), frequência cardíaca pico $\left(\mathrm{FC}_{\text {pico }}\right)$ e percepção subjetiva de esforço pico $\left(\mathrm{PSE}_{\text {pico }}\right)$

\begin{tabular}{|c|c|c|c|c|}
\hline Variáveis & AT $(n=\mid 5)$ & $\mathrm{GF}(n=8)$ & $\mathrm{GB}(\mathrm{n}=7)$ & $P$ \\
\hline$P A n_{\text {med }}(W)$ & $479,6 \pm 108,0$ & $468,5 \pm 104,2$ & $492,6 \pm 119,0$ & 0,678 \\
\hline$P A n_{\max }(W)$ & $633,6 \pm 144,3$ & $625,3 \pm 107,0$ & $643,1 \pm 171,9$ & 0,822 \\
\hline $\mathrm{T}(\mathrm{s})$ & $38,5 \pm 4,1$ & $39,7 \pm 4,4$ & $35,9 \pm 2,8$ & 0,069 \\
\hline $\mathrm{IFI}(\mathrm{W} / \mathrm{s})$ & $6,8 \pm 3,3$ & $6,6 \pm 3,5$ & $7,1 \pm 3,3$ & 0,774 \\
\hline IF2 (\%) & $39,2 \pm 14,9$ & $40,6 \pm 19,1$ & $37,6 \pm 9,4$ & 0,057 \\
\hline $\mathrm{Lac}_{\text {pico }}(\mathrm{mM})$ & $7,8 \pm 1,1$ & $8,1 \pm 1,5$ & $7,4 \pm 0,4$ & 0,265 \\
\hline $\mathrm{FC}_{\text {pico }}(\mathrm{bpm})$ & $181,7 \pm 11,4$ & $184,6 \pm 12,4$ & $178,3 \pm 9,9$ & 0,553 \\
\hline $\mathrm{PSE}_{\text {pico }}$ & $14,6 \pm 3,0$ & $15,7 \pm 3,3$ & $13,3 \pm 2,1$ & 0,211 \\
\hline
\end{tabular}

Para GF, os valores das variáveis antropométricas e de composição corporal tiveram efeito direto na PSE pico $_{\text {e no tempo total do RAST. A PSE }}$ pico correlacionou-se significativamente à massa corporal $(r=0,90)$, massa gorda $(r=0,85)$, massa isenta de gordura $(r=0,83)$, circunferência de coxa $(r=0,91)$, peitoral $(r=0,79)$, cintura $(r=0,8 \mathrm{I})$ e abdome $(r=0,8 \mathrm{I})$; já o tempo total apresentou correlação com a massa corporal $(r=0,75)$, massa gorda $(r=0,73)$, circunferência de cintura $(r=$ $0,72)$, abdome $(r=0,75)$ e coxa $(r=0,84)$. Além disso, em GF, maiores potências 
afetam negativamente o tempo de teste e o índice de fadiga, com potência média correlacionando-se negativamente ao tempo de teste $(r=-0,85)$ e potência pico positivamente ao índice de fadiga absoluto $(r=0,77)$.

Já para GB, a massa corporal e a massa isenta de gordura favorecem as variáveis relacionadas ao teste RAST enquanto que a massa gorda as influencia negativamente. A massa corporal correlacionou-se somente com $\mathrm{FC}_{\text {pico }}(r=0,79)$, a massa gorda com Lac pico $(r=0,76)$, potência pico $(r=-0,78)$, tempo total do RAST $(r=0,79)$ e índice de fadiga $(r=-0,94)$, a massa isenta de gordura com a potência média $(r=0,80)$ e pico $(r=0,77)$, a estatura com Lac pico $_{(}(r=-0,82)$ e índice de fadiga $(0,84)$, e a circunferência de abdome com Lac $c_{\text {pico }}(r=0,8 \mathrm{I})$ e índice de fadiga $(r=-0,78)$. Neste grupo também houve correlação negativa entre $L A c_{\text {pico }}$ e índice de fadiga $(r=-0,93)$, e positiva entre índice de fadiga, potência pico $(r=0,88)$ e média $(r=0,76)$.

\section{DISCUSSÃO}

O objetivo do presente estudo foi determinar alguns indicadores antropométricos e fisiológicos de uma amostra de atletas de rugby no Brasil, comparando-se essas características entre as posições forwards e backs. Os principais achados evidenciam a influência da composição corporal e indicadores fisiológicos na potência anaeróbia de atletas de rugby e, sendo este um fator determinante na performance desta modalidade, verifica-se que a equipe estudada não utilizou tais critérios para seleção de atletas e discriminação de posições de jogo.

GF apresentou valores significativamente maiores para as variáveis antropométricas quando comparado à $\mathrm{GB}$, com exceção da estatura. A massa corporal média encontrada em jogadores de rugby amadores e profissionais fica em torno de 86 e 90 kg (GABBETT, 2002; GABBETT, 2000), valores menores do que encontrado no presente estudo para GF e para a amostra total. Considerando-se o IMC, também é possível verificar que GB está classificado como sobrepesado (IMC = 25 - 29,9) e GF como obeso grau I (IMC = 30 - 34,9) (WORLD, 1982), além de apresentarem valores de \%G consideravelmente superiores aos vistos na literatura por Crewther et al. (2009) (forwards = 14,4 $\pm 3,0 \%$; backs $=10,3 \pm 1,9 \%$ ) e Brewer, Davis e Kear ( 1994) (forwards = 15,2 \%; backs = 12,6\%)

De fato, a literatura apresenta os forwards como jogadores de maior tamanho corporal (SCOTT et al., 2003; NICHOLAS; BAKER, 1995), porém, ao contrário do que foi verificado no presente estudo, maiores valores de massa corporal e circunferências não deveriam ser relacionados à maior massa gorda. A intermitência de esforços observada durante uma partida de rugby faz com que os forwards 
percorram maiores distâncias em alta intensidade, quando comparados aos backs (COUTTS; REABURN; ABT, 2003; SCOTT et al., 2003; DEUTSCH et al., 1998; MEIR; ARTHUR; FORREST, 1993), e, como são jogadores que dependem também de uma maior massa corporal, menores valores de $\% \mathrm{G}$ representariam um menor dispêndio energético para sustentar a carga extra e não funcional de gordura durante os deslocamentos, aprimorando a potência, velocidade e eficiência durante todo o jogo (MARX et al., 200I).

Crewther et al. (2009) apontou que, apesar da expressão de potência de membros inferiores ser altamente correlacionada com o tamanho corporal em jogadores de elite, somente o treinamento com vistas ao aumento do tecido muscular poderia aumentar o rendimento neste parâmetro. Isto fica explícito no presente estudo, já que em GB a massa corporal e a MIG influenciaram positivamente as variáveis do teste RAST e a massa gorda negativamente, com poder de explicação da massa gorda ao aumento do tempo total do teste RAST de 53\% para GF e $62 \%$ para GB, além de explicar piores valores de índice de fadiga de GB em 88\%.

Olds (200 I) verificou, ao longo dos anos, em jogadores de rugby, uma tendência gradual de modificação do perfil endomorfo para mesomorfo, em especial para os forwards. Nota-se que a equipe do presente estudo ainda está distribuída perante padrões antigos, sem direcionamento de aspectos antropométricos que favoreçam melhores performances.

De forma geral, os critérios empíricos que ainda motivam a disposição dos sujeitos de maior tamanho corporal e \%G para a posição de forwards se resumem na maior capacidade de suportar e efetuar movimentos de contato, e um provável menor risco de lesão nos impactos (MEIR; ARTHUR; FORREST, 1993). A seleção de atletas começa a apresentar problemas quando esses são os únicos fatores considerados. Estudos com equipes profissionais e de elite demonstram, como no presente estudo, valores significativamente maiores de massa corporal e \%G para os forwards comparados aos backs, no entanto, diferenças também são verificadas na capacidade de sprint, potência pico e média, força máxima e capacidade aeróbia (CREWTHER et al., 2009; MEIR et al., 200 I).

No presente estudo, nenhuma diferença é vista entre os grupos no teste RAST e nas variáveis fisiológicas resultantes do mesmo, o que seria esperado, em função das características distintas de cada posição durante o jogo (GABBETT, 2002), já que o RAST simula claramente os esforços de alta intensidade decisivos no rugby e a capacidade do atleta em recuperar-se rapidamente para o esforço seguinte (índice de fadiga).

Os deslocamentos rápidos e potentes para recuperação da bola ou avanço no ataque são efetuados com maior frequência pelo forwards (SCOTT et al., 2003; 
DEUTSCH et al., 1998), e esses não demonstraram ser superiores aos backs nas análises efetuadas. Para os backs, alta velocidade é requerida para condução de bola, marcação do ponto e esquiva dos defensores (DUTHIE; PYNE; HOOPER, 2003), e estes também não se destacaram nestas capacidades, já que o tempo de execução e consequentemente a velocidade de corrida no RAST não foram diferentes entre os grupos.

Além disso, sabendo-se que a razão de trabalho intenso/leve dos forwards indica maior quantidade de esforço destes durante uma partida em relação aos backs (DEUTSCH et al., 1998; MEIR; ARTHUR; FORREST, 1993), uma melhor capacidade de recuperação representaria maior possibilidade de manutenção de ótimas performances; no entanto, não houve diferença entre GF e GB para o índice de fadiga no teste de RAST. Esta variável apresentou alta correlação com as variáveis de potência anaeróbia pico e média de ambos os grupos, mostrando que os atletas avaliados não conseguem manter, por longos períodos, altas intensidades em exercícios intermitentes.

Crewther et al. (2009) encontraram em jogadores de elite de rugby, diferenças relacionadas às demandas específicas de potência, velocidade e capacidade de recuperação dos atletas pertencentes à posição forward ou back, atribuindo a isso a correta seleção de atletas e, principalmente, especificidade de treinamento.

Em relação aos índices fisiológicos, Coutts; Reaburn e Abt (2003) mostram que os valores de lactato reduzem significativamente entre o primeiro $(8,4 \mathrm{mM})$ e segundo tempo (5,9 mM), sendo os valores do primeiro tempo maiores que os de $\mathrm{Lac}_{\text {pico }}$ da amostra total do presente estudo. Provavelmente a fadiga acumulada impeça a manutenção de esforços de alta intensidade que favoreçam o acúmulo de lactato sanguíneo, fato que pode ser comparado ao alto índice de fadiga apresentado pelos sujeitos do presente estudo durante os seis sprints do teste RAST.

Comparando-se posições, os valores de lactato sanguíneo reportados na literatura são similares aos do presente estudo, com valores médios de jogo de 6,6 e 5, I mM (DEUTSCH et al., 1998), e 8,5 e 6,5mM (COUTTS; REABURN; ABT, 2003), respectivamente para forwards e backs. Essa maior capacidade de produção de lactato representou para GB um menor índice de fadiga $(r=-0,93)$, o que não está relacionado à maior intensidade de esforço, uma vez que maiores valores de potência pico representaram maiores índices de fadiga $(r=0,88)$. Aparentemente, os jogadores com maior capacidade de produção de energia por vias glicolíticas conseguem manter altas intensidades por mais tempo.

Diante disso, Atkins (2006) verificou diferenças significativas na concentração de lactato sanguíneo após teste de esforço máximo entre jogadores profissionais ( I 0,8 mM) e semiprofissionais (9, I mM), mostrando que provavelmente o nível de 
treinamento influi nessas variáveis, já que os atletas amadores do presente estudo apresentaram valores ainda menores.

$A F C_{\text {pico }}$ da amostra total do presente estudo ( 181 bpm) demonstrou-se levemente menor às reportadas em estudos com análise de jogo ( 89 bpm) (GABBETT, 2002) e durante teste de esforço ( 191 bpm) (ATKINS, 2006), mostrando que provavelmente o menor nível de treinamento impeça a execução de esforços máximos, demonstrados por baixas PSE $_{\text {pico }}$ após seis sprints em velocidades teoricamente máximas.

Essas variáveis apresentaram algumas correlações com variáveis antropométricas em ambos os grupos. Em GF, variáveis relacionadas ao tamanho corporal, como circunferências e massa corporal, apresentaram poder de explicação de até

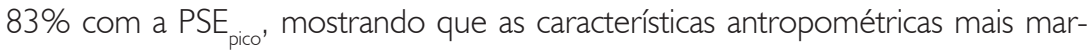
cantes deste grupo aumentam o esforço percebido durante exercícios máximos e consequentemente podem prejudicar a performance. Em GB, a massa corporal apresentou poder de explicação de $62 \%$ à $\mathrm{FC}_{\text {pico' }}$ mostrando que para esse grupo, a situação de sobrepeso (IMC = 25,9 \pm 2,5) aumenta o esforço cardíaco e também pode acarretar piores performances durante um jogo.

Reforça-se que a equipe estudada não representa a realidade de todas as equipes do país, entretanto se aproxima do contexto da grande maioria dos pequenos centros de treinamento de rugby que podem revelar atletas, como aconteceu com um dos avaliados do presente estudo, convocado para a seleção brasileira. O baixo número de praticantes envolvidos em um treinamento sistematizado no Brasil dificulta a seleção de grandes amostras, no entanto ressalta-se a importância de avaliar equipes completas, com as diversas subdivisões das posições utilizadas no presente estudo. Uma limitação do presente estudo foi a utilização do protocolo de dobras cutâneas para determinação do \%G em sujeitos que, mesmo sendo atletas, encontravam-se em grau de obesidade, entretanto, ainda assim foi possível ressaltar os erros na seleção de atletas e determinação do posicionamento de jogo utilizando critérios empíricos e visuais, como o tamanho corporal.

\section{CONCLUSÃO}

Em suma, o presente estudo verificou que, para a equipe estudada, a determinação das posições de jogo ocorre prioritariamente em função das características antropométricas dos atletas, entretanto, os resultados demonstraram que a potência anaeróbia, característica de suma importância no rugby, é fortemente influenciada pela composição corporal e por variáveis fisiológicas. Diante disso, sugere-se que as necessidades inerentes às posições específicas da modalidade sejam respeitadas na seleção, controle e prescrição de treino de atletas de rugby, sendo avaliadas a partir de testes físicos e antropométricos. 


\section{Anthropometrical and Physiological Parameters of Brazilian Rugby Athletes Sample}

ABSTRACT: We examined physiological and anthropometrical parameters of rugby players. Fifteen athletes, grouped according to game position: forwards (GF) and backs (GB), underwent anthropometric assessment and anaerobic power test with blood lactate analysis, heart rate and perceived exertion measures. The Student $t$ test showed differences between groups for anthropometrical variables, particularly for body mass ( $G F=102.6 \pm 16.0 \mathrm{~kg} ; G B=80.0$ $\pm 6.8 \mathrm{~kg}$ ) and fat mass $(G F=28.0 \pm 10.6 \mathrm{~kg} ; G B=13.8 \pm 5.6 \mathrm{~kg})$, but no differences for variables measured in the power test. Furthermore, the Pearson correlation test showed negative influence of fat mass in test time for GF $(r=0.73)$ and positive of fat-free mass in power for $G B(r=0.80)$, highlighting that body composition influences rugby performance and should be used with physiological parameters for position determinations.

KEYWORDS: Energetic Metabolism; Lactate; Body Composition; Running.

\section{Los indicadores antropométricos y fisiológicos de una muestra brasileña de atletas de rugby}

RESUMEN: Hemos examinado indicadores fisiológicos y antropométricos de jugadores de rugby. Quince atletas, agrupados de acuerdo con la posición de juego: hacia delante (GF) y espalda (GB) realizaron evaluación antropométrica y test de potencia anaeróbica con análisis de lactato sanguíneo, frecuencia cardíaca y el esfuerzo percibido. El test t de Student mostró diferencias entre los grupos para las variables antropométricas, en particular de masa corporal ( $G F=102,6 \pm 16,0 \mathrm{~kg} ; G B=80,0 \pm 6,8 \mathrm{~kg}$ ) y la masa grasa $(G F=28,0 \pm 10,6 \mathrm{~kg}$; $G B=13,8 \pm 5,6 \mathrm{~kg})$, pero no para las variables medidas en el test de potencia. Además, el test de correlación de Pearson mostró influencia negativa de la masa grasa en el tiempo de test para $G F(r=0,73)$ y positiva para la masa libre de grasa en la potencia para $G B(r=0,80)$, resaltando que la composición corporal influye en el desempeño en el rugby, y se debe utilizar en conjunto con los parámetros fisiológicos para discriminar las posiciones en este deporte. PALABRAS CLAVE: Metabolismo energético; lactato; composición corporal; carrera.

\section{REFERÊNCIAS}

ATKINS, S. J. Performance of the yo-yo intermittent recovery test by elite professional and semiprofessional rugby league players. Journal of Strength and Conditioning Research, Colorado Springs, v. 20, n. I, p. 222-225, feb. 2006.

BORG, G. A. Psychophysical bases of perceived exertion. Medicine and Science in Sports and Exercise, Hargestown, v. 14, n. 5, p. 377-381, 1982.

BREWER, J.; DAVIS, J.; KEAR, J. A comparion of the physiological characterístics of rugby league forwards and back. Journal of Sports Science, London, v. 12, n. 2, p. I 58- I62, feb. 1994.

CONFEDERAÇÃO BRASILEIRA DE RUGBY. (CBRu) Rugby no âmbito nacional. 20। I. Disponível em: <http://www.brasilrugby.com.br>. Acesso em: 12 fev. 201 I. 
COMITÊ OLÍMPICO INTERNACIONAL (COI). 12 I $^{\text {a }}$ sessão do COI. Copenhagem, 2009.

COUTTS, A.; REABURN, P.; ABT, G. Heart rate, blood lactate concentration and estimated energy expenditure in a semi-professional rugby league team during a match: a case study. Journal of Sports Science, London, v. 21, n. 2, p. 97-103, feb. 2003.

CREWTHER, B. T. et al. Neuromuscular performance of elite rugby union players and relationships with salivary hormones. Journal of Strength and Conditioning Research, Colorado Springs, v. 23, n. 7, p. 2046-2053, oct. 2009.

DEUTSCH, M. U. et al. Heart rate, blood lactate and kinematic data of elite colts (under-19) rugby union players during competition. Journal of Sports Science, London, v. I6, n. 6, p. 561-570, aug. 1998.

DUTHIE, G.; PYNE, D.; HOOPER, S. Applied physiology and game analysis of rugby union. Sports Medicine, Auckland, v. 33, n. 13, p. 973-991, nov. 2003.

GABBETT, T. J. Science of rugby league football: a review. Journal of Sports Science, London, v. 23, n. 9, p. 96।-976, sept. 2005.

GABBETT, T. J. Influence of physiological characteristics on selection in a semi-professional first grade rugby league team: a case study. Journal of Sports Science, London, v. 20, n. 5, p. 399-405, may 2002.

GABBETT, T. J. Physiological and anthropometric characteristics of amateur rugby league players. British Journal of Sports Medicine, Loughborough, v. 34, n. 4, p. 303-307, aug. 2000.

JACKSON, A. S.; POLLOCK, M. L. Generalized equations for predicting body density of men. British Journal of Nutrition, Cambridge, v. 40, n. 3, p. 497-504, nov. 1978.

MARX, J. O. et al. Low-volume circuit versus high-volume periodized resistance training in women. Medicine and Science in Sports and Exercise, Hagerstown, v. 33, n. 4, p. 635- 643, apr. 2001 .

MEIR, R. A.; HALLIDAY, A. J. Pre- and post-game body mass changes during an international rugby tournament: a practical perspective. Journal of Strength and Conditioning Research, Colorado Springs, v. 19, n. 3, p. 7|3-7|6, aug. 2005.

MEIR, R. et al. Physical fitness qualities of professional rugby league football players: determination of positional differences. Journal of Strength and Conditioning Research, Colorado Springs, v. 15, n. 4, p. 450-458, nov. 2001.

MEIR, R.; ARTHUR, D.; FORREST, M. Time and motion analysis of professional rugby league: A case study. Strength and Conditioning Coach, Colorado Springs, n. I, p. 24-29, feb. 1993.

NICHOLAS, C. W.; BAKER, J. S. Anthropometric and physiological characteristics of first- and second-class rugby union players. Journal of Sports Science, London, v. I, n. 3, p. I3- I 5, feb. 1995. 
OLDS, T. The evolution of physique in male rugby union players in the twentieth century. Journal of Sports Science, London, v. 19, n. 4, p. 253-262, apr. 200 I.

SCOTT, A. C. et al. Aerobic exercise physiology in a professional rugby union team. International Journal of Cardiology, Maryland Heights, v. 87, n. 2/3, p. 173-177, feb. 2003.

SIRI, W. E. Tecniques for measuring body composition. Washington: National Academy, I96I.

WORLD HEALTH ORGANIZATION (WHO). Obesity: preventing and managing the global epidemic. Geneva, 1998.

ZACHAROGIANNIS, E.; PARADISIS, G.; TZIORTZIS, S. An evaluation of tests of anaerobic power and capacity. Medicine and Science in Sports and Exercise, Hagerstown, v. 36, s। I 6, 2004.

ZAGATTO, A. M.; BECK, W. R.; GOBATTO, C. A. Validity of the running anaerobic sprint test for assessing anaerobic power and predicting short-distance performances. Journal of Strength and Conditioning Research, Colorado, v. 23, n. 6, p. I820-1827, sept. 2009.

Recebido em: 17 jan. 2012 Aprovado em: 18 dez. 2012

Endereço para correspondência:

Fabiana Andrade Machado Depto. Educação Física, Bloco M06 - UEM Av. Colombo, 5.790 - Jd. Universitário Maringá-PR 